\title{
The Prevalence of Inappropriate Use of Over the Counter NSAIDS Purchased in Community Pharmacies in Western Australia
}

\author{
Ahmad Alzayadi* \\ Curtin University, Australia \\ *Corresponding author: Ahmad Frahan Khinteel Alzayadi, Ph.D, Curtin University, Bentley, Australia \\ Submission: 悳 October 17, 2017; Published: 眥 November 16, 2017
}

\section{Introduction}

The use of over-the-counter (OTC), non-steroidal antiinflammatory drugs (NSAIDs) has become commonplace in the western world. NSAIDs increase the risk of heart failure in elderly patients as well as other thrombotic events [1]. This has led the Food and Drug Administration (FDA) in the United States of America to report that the use of non-selective NSAIDs potentially increases cardiovascular risks [2]. In Australia, cardiovascular disease is one of the largest health problems, affecting more than 3.4 million Australians [3]. A large Australian study of 3569 subjects, $1492(41.8 \%)$ who reported an upper GI problem had used NSAIDs [4]. Further, NSAIDs alone or in combination with diuretics and/or ACE inhibitors were found to be involved in over $50 \%$ of iatrogenic acute renal failure cases [5]. These data stress the need for precautions with the use of NSAIDs especially in people with risk factors. Owing to reasons, such as the availability of the OTC NSAIDs for self-care of many health conditions and the limited knowledge of NSAIDs by purchasers. There is potential that NSAIDs are inappropriately used. Two American surveys were conducted to identify patterns of use and public perceptions of OTC pain relievers. One with more than 9000 respondents reported that participants using OTC NSAIDs exclusively, $60 \%$ were unaware of the risks of side effects from NSAIDs and approximately $30 \%$ did not believe they were at risk [6]. A second survey conducted in 2002 found that approximately 50\% of the participants were not worried about possible side effects [6]. This survey also indicated that $30 \%$ believed there were few risks associated with taking OTC NSAIDs.

Inappropriate use of drugs is associated with increased adverse drug events, hospitalization and mortality [7]. Studies indicate that nearly $32 \%$ of patients who filled 3 or more NSAID prescription used NSAIDs inappropriately, including consuming two or more NSAIDs, exceeding the recommended daily dosage or both [8]. Of 1,800 Australians in questioner in $2009,85 \%$ had used OTC painkillers, $15 \%$ of those indicated they were taking an NSAID although they had a health condition that could be exacerbated by the NSAID, or that there was a possible harmful interaction with another medication that they were prescribed [9]. Multiple studies have documented the prevalence of inappropriate NSAIDs use $[6,10-12]$. These estimate that $7 \%$ of frequent monthly painkiller users reported using two or more analgesics nearly every day during the month. Another Australian survey conducted in 2010 of 1004 participants, found that four in five people were totally unaware of the heart risks posed by NSAIDs. In addition, one in two of those currently taking an NSAID had been diagnosed with heart disease or stroke, or had a direct family member who was living with such a condition [13]. To our knowledge, there is no study that describes the prevalence of inappropriate use of OTC NSAIDs in the suburbs of Perth, Western Australia.

\section{Aim of the study}

The objective of the study was to elucidate the prevalence of inappropriate use of OTC NSAIDs purchased at community pharmacies in suburban Perth. Additionally, to investigate the level of knowledge that purchasers had of the safety level of OTC NSAIDs and to record the findings, recommendations and the outcomes of the pharmacist's interventions after inappropriate use has been identified.

\section{Ethics approval}

This study was approved by the Human Ethics Committee of Curtin University in accordance with the National Health and Medical Research Council's guidelines (approval number $\mathrm{PH} / 14 / 2010)$.

\section{Methods}

\section{Data collection}

Five pharmacy owners in the suburban areas of Balga, Mirrabooka, Marangaroo Mt Lawley and Gosnells agreed to take part in the study. They were given an information sheet that explained the study details and reassured them that their customers will be treated with respect at all times. The pharmacists on duty at these pharmacies were asked to recruit participants for the study. Inclusion criteria were customers who over 18 years of age, who asked for an NSAID for themselves and who consented to involve 
in the survey. They were given an information sheet to explain the nature of the survey and assure of the anonymity. The survey was conducted in the form of interviews with questions taken from a questionnaire written by the researcher (available online). Only pharmacists conducted the survey. Most interviews took no longer than ten minutes. As participants gave their answers, pharmacists ticked the relevant boxes on the questionnaire. At the time of the interview, if the pharmacist conducting the survey identified a problem, a recommendation was made and this was recorded. After the data has been collected, the pharmacists' interventions were analysed. For this purpose, a modified check list amended from the "DOCUMENT" used in the Promise phase III study was used [14]. This checklist, see Table 1, allowed for problems related to drug selection, dosing, compliance, under-treatment or under-treated indication to be identified. If any of the boxes in this drug related problem checklist were ticked, then the participants were deemed to be using the OTC NSAIDs inappropriately (Table 1).

Table 1: Checklist for finding drug-related problems.

\begin{tabular}{|l|}
\hline Drug related problem \\
\hline Drug selection: \\
. Dose change \\
.Inappropriate dosage form \\
.Drug interaction \\
.Contraindication apparent \\
.Wrong drug \\
.No indication apparent \\
.Other drug selection problem \\
\hline Over or under-dose: \\
. High dose \\
. Incorrect or unclear dosing instructions \\
. Low dose \\
. Other dose problem \\
\hline Compliance: \\
. Taking too little \\
. Intentional drug misuse \\
. Taking too much \\
. Other compliance problems \\
\hline Under treated or under treated: \\
. Condition not adequately treated indication \\
. Preventative therapy required \\
. Other undertreated indication problem \\
\hline Recommendation \\
\hline A change in therapy: \\
. Dose change \\
. Dose frequency change \\
. Drug change \\
. Medicine not dispensed \\
\hline
\end{tabular}

\begin{tabular}{|l|}
\hline A referral required: \\
. Refer to prescriber \\
. Refer for medication review \\
. Refer to hospital \\
.Other referral required \\
\hline Provision of information: \\
. Education or counseling \\
. Commence dose administration aid \\
. Written summary of medications \\
. Other written information \\
\hline
\end{tabular}

\section{Data analysis}

Use of OTC NSAIDs was deemed to be inappropriate if the pharmacist conducting the survey did any one or more of the following:

1. Change of the requested NSAID to a different medicine;

2. Referred the customer to their doctor;

3. Refuse of the sale of the requested NSAID

If the participants had any health condition(s), such as hypertension and peptic ulcer disease, that contraindicate the use of NSAIDs but they were monitored by their doctor, then they were not deemed to be using the NSAID inappropriately. Figure 1 provides a schematic summary of the study (Figure 1).

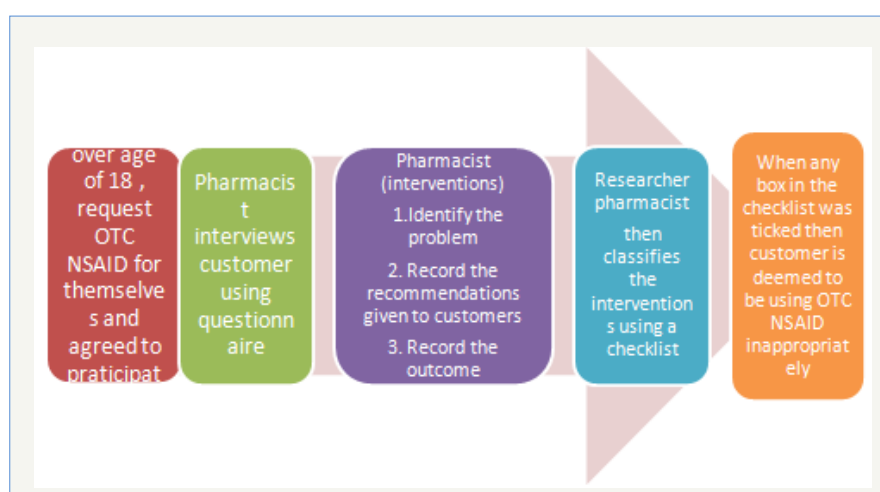

Figure 1: Schematic summary of the study.

The Pharmaceutical Society of Australia Professional Practice Standards include a standard on Counseling, which reads: "The pharmacist works with the consumer to provide tailored verbal and written information to ensure the consumer has sufficient knowledge and understanding of their medicines and therapeutic devices to facilitate safe and effective use" [15]. This provided the basis for the study and if a pharmacist suspects potential contraindications to OTC NSAID use, then the pharmacist is professionally bound to intervene. For instance, in one case, a patient was on methotrexate, indomethacin and requested Ibuprofen +Codeine. This patient had a GI bleed at that time as evidenced by the passing of dark stools. In this case an immediate referral was recommended by the pharmacist. In another example, where an immediate referral is not necessary, is when a number of patients come into the pharmacy 
asking for Ibuprofen to control their dental pain. Pharmacist in this case would sell the product and inform the patient to see his/her dentist in a week's time or so. In the latter example, it was not clear if this case should be classified as a referral or not when utilizing the modified DOCUMENT classification. For this study, we classified this case as a "non-referral" case.

\section{Statistical analysis}

A sample size of 200 participants was designed to give adequate precision to the prevalence estimate. A sample of size 196 would lead to a 95\% Confidence Interval for an accuracy of $\pm 7 \%$ at the most, when the prevalence is $50 \%$. If the prevalence is lower or higher than $50 \%$, the precision would be unproved (narrower confidence interval). The secondary objective to identify factors associated with inappropriate use, and, while it is difficult to be precise on a sample size which would be adequate for this objective, a general "rule of thumb" is that a sample of size at least 120 should be adequate to identify variables demonstrating a 'moderate' association [16]. The data recorded on the forms were summarized using standard descriptive statistics -frequencies for categorical variables and means, standard deviations and medians for variables measured on a continuous scale. The proportion of recruited subjects who were classified as 'Inappropriate Use' was calculated along with its $95 \%$ confidence interval using standard methods.

A Logistic Regression model was used to identify any subject characteristics (age, gender, medical history, etc.) which could be independently associated with inappropriate use. The results from this analysis are presented with their p-values. A p-value $<0.05$ was interpreted as indicating a statistically significant association. The subject knowledge of appropriate conditions for NSAID use are summarized (frequencies) and areas where knowledge was poor are highlighted.

\section{Results and Discussion}

\section{Demographics}

Demographic characteristics of participants in the survey presented in (Figure 2).

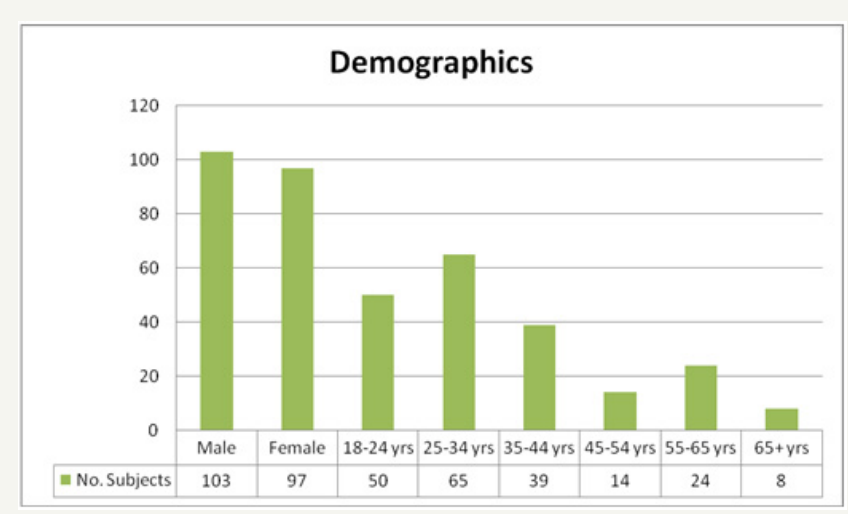

Figure 2: Demographics of participants.

A hundred and three are females (51.5\%) and 97 are males (48.5\%). The majority of participants are 25-34 years of age
(32.5\%), followed by $25 \%$ who are $18-25$ years old, $19.5 \%$ who are $35-44$ years old, $12 \%$ who are $55-64$ years old, while $7 \%$ are $45-54$ years old and $4 \%$ are over 65 years old. The genders were approximately similar however a majority was less than 35 years. The most requests were for ibuprofen plus codeine, with ibuprofen the most requested NSAIDs. Figure 3 summaries the type of OTC NSAIDs requested by participants (Figure 3).

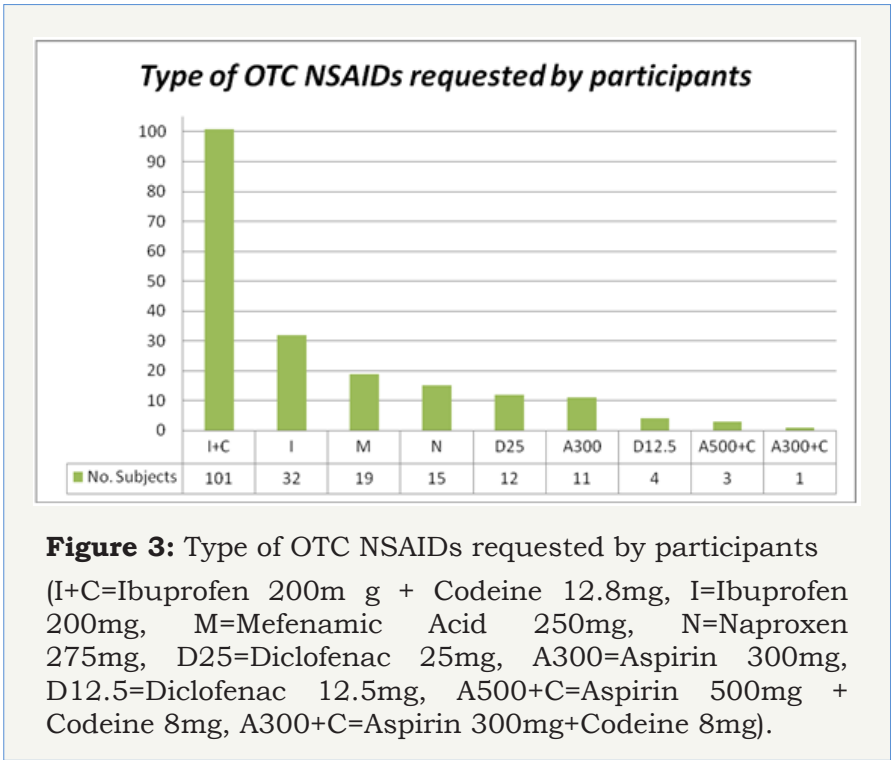

101 of the participants requested ibuprofen +Codeine (50.25\%), 32 requested ibuprofen (16\%) , 19 requested mefenamic acid $(9.55 \%)$, while 15 requested naproxen $(7.54 \%)$ and 12 wanted diclofenac $25 \mathrm{mg}$ (6.03\%). Medical reasons for requested OTC NSAIDs Medical conditions, for which OTC NSAIDs were requested for, are presented in (Figure 4).

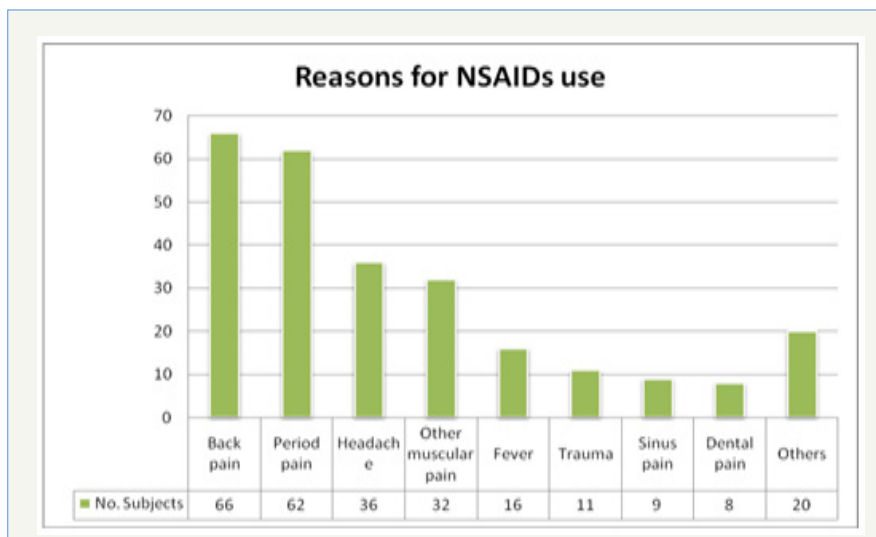

Figure 4: Medical reasons for requested OTC NSAIDs.

Of the 200 participants, 66 were using an NSAID for back pain, while 62 were taking it for period pain and 36 were taking it for headache. Others were taking it for a variety of reasons such as muscular pain, fever and trauma. Of the total concurrent health conditions found among participants (118), 32 had hypertension (27.1\%) and 20 were asthmatic (16.9\%). More concurrent medical conditions are presented in (Figure 5).

Of the 200 respondents, 87 had never used the NSAID requested (43.5\%) compared to 113 who have used the requested 
NSAID (56.5\%). Of the latter, 60 were not taking the NSAID at present (53.1\%), while 53 were current users (46.9\%). Amongst current users, 36 had been using NSAIDs for more than a month $(67.92 \%)$ i.e. long term users, while 17 used NSAIDs for a month or less $(32.08 \%)$ i.e. short term users. All of the above are presented in (Figure 6).

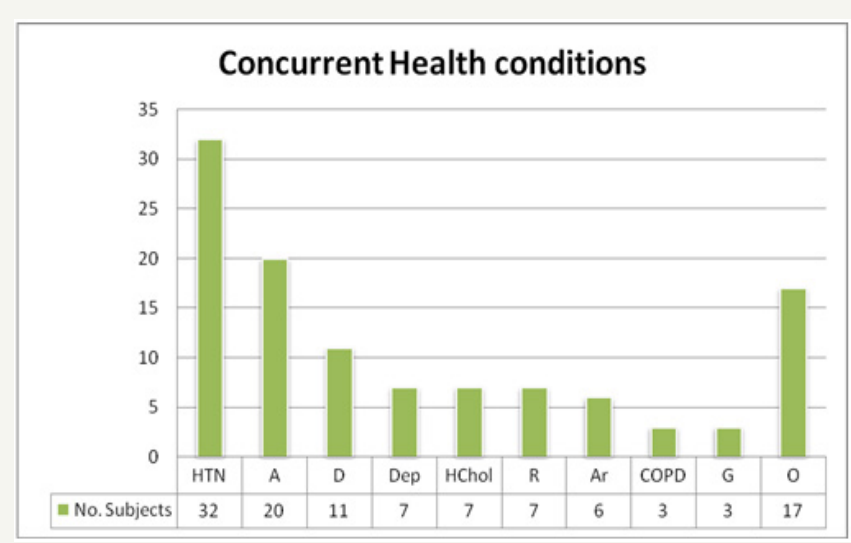

Figure 5: Concurrent health conditions of participants (HTN: Hypertension, A: Asthma, D: Diabetes, Dep: Depression, Hchol: High Cholesterol, R: Reflux, Arth: Arthritis, COPD: Chronic Obstructive pulmonary disease, G: Gout and O: Others).

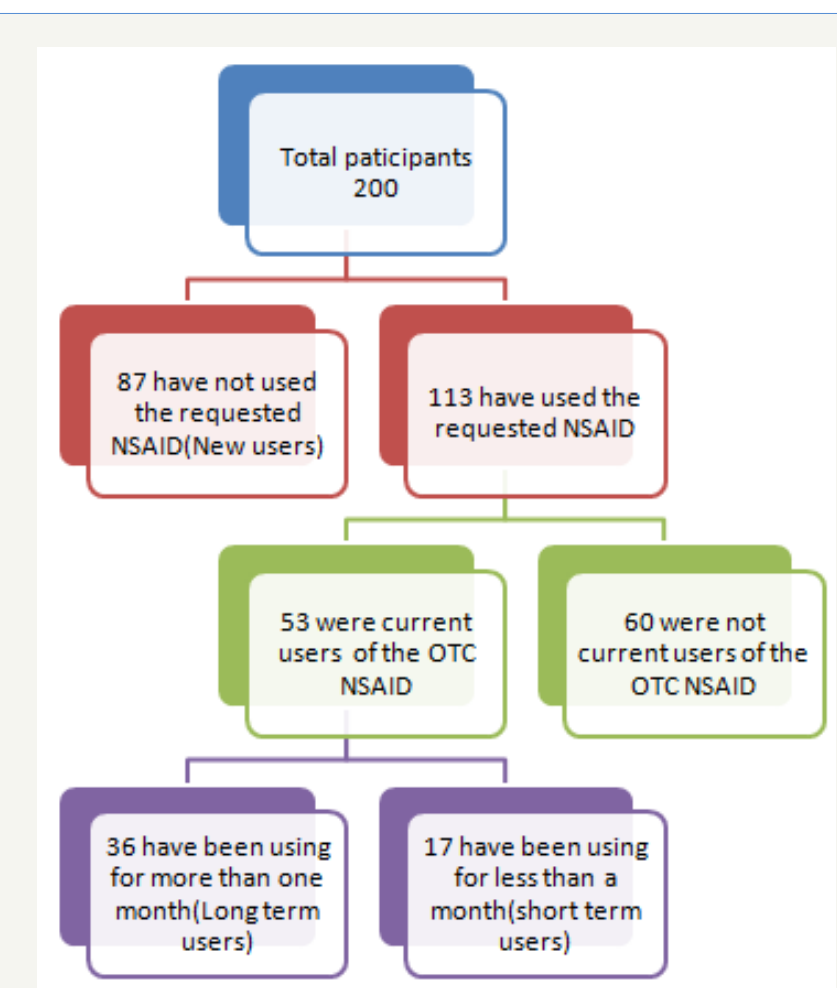

Figure 6: Long term, short term and new users of OTC NSAIDs.

Of the long term users $(n=36), 20$ were taking ibuprofen + Codeine and 8 of them were taking ibuprofen. There was no significant difference between the gender of long term users i.e. 21 females and 15 males $(p=0.25)$. The two main age groups were 35-44 years corresponding to 13 people and 10 in the 25-34 years old group. Twenty four of the 36 long term users were taking the OTC NSAIDs for back pain (66.7\%), while only 3 of 17 short term users were taking the OTC NSAIDs for back pain $(p=0.0009)$. Other parameters (such as type of medicine requested, patient's parameters, recommendations, concurrent health conditions and buying NSAIDs from other places) show no statistical difference between long term users and short term users ( $p>0.05$ ). Approximately a quarter of the long term users (22\%) were on anti-hypertensive medications and $17 \%$ were taking anti-asthmatic medications, see (Figure 7).

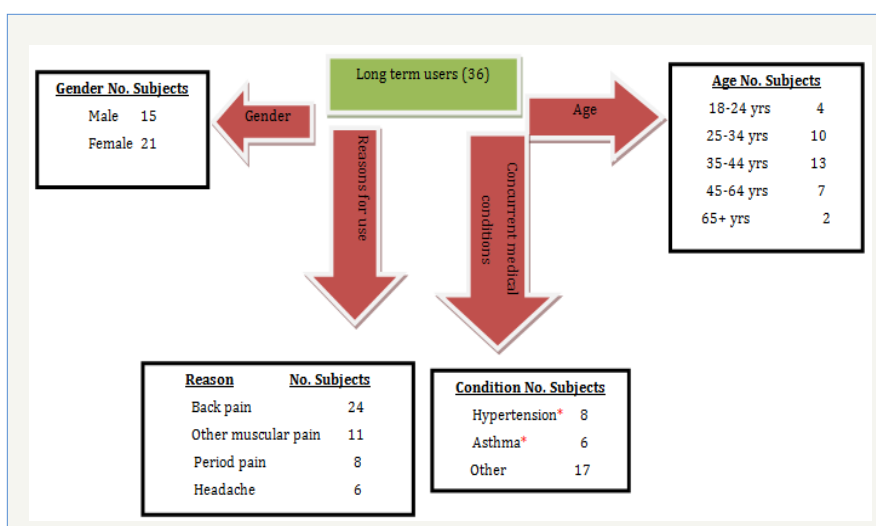

Figure 7: Demographics of long term users of requested NSAIDs.

*Subjects have medical conditions as well as medications for these medical conditions i.e. anti-hypertensive and antiasthmatic medications.

Using the checklist for identifying drug related problems, $55.6 \%$ of the long term users were found to be using NSAID inappropriately see (Table 2).

Table 2: Prevalence of inappropriate OTC NSAIDs use in different categories.

\begin{tabular}{|c|c|}
\hline $\begin{array}{c}\text { Characteristics No. of patients with } \\
\text { inappropriate medication use (\%) }\end{array}$ & $\begin{array}{c}\text { Number of subjects } \\
\text { (\%)(n=200) }\end{array}$ \\
\hline Male 31 (32\%) & $97(48.5 \%)$ \\
\hline Female 25 (24\%) & $103(51.5 \%)$ \\
\hline Age (years) & \\
\hline $18-245(10 \%)$ & $50(25 \%)$ \\
\hline $25-3411(17 \%)$ & $65(32.5 \%)$ \\
\hline $35-4413(33 \%)$ & $39(19.5 \%)$ \\
\hline $45-546(43 \%)$ & $14(7 \%)$ \\
\hline $55-6415(63 \%)$ & $24(12 \%)$ \\
\hline $65+6(75 \%)$ & $8(4 \%)$ \\
\hline Chronic Health conditions: & $31(15.5 \%)$ \\
\hline Hypertension \& Asthma 3 (100\%) & \\
\hline Hypertension (no asthma) 21 (67.7\%) & $31.5 \%)$ \\
\hline
\end{tabular}




\begin{tabular}{|c|c|}
\hline Asthma (no hypertension) $12(70.6 \%)$ & $17(8.5 \%)$ \\
\hline Other condition(s)* $7(23.3 \%)$ & $30(15 \%)$ \\
\hline None $12(10 \%)$ & $119(59.5 \%)$ \\
\hline New users of requested NSAID $15(17.2 \%)$ & $87(43.5 \%)$ \\
\hline Had NSAID before $40(35.4 \%)$ & $113(56.5)$ \\
\hline Long term users of requested $20(55.6 \%)$ & $36(18 \%)$ \\
\hline NSAID & \\
\hline
\end{tabular}

Source of recommended NSAIDs to participants

Of the 200 participants, 119 said the NSAID was recommended by their doctor $(59.5 \%)$ and 30 reported that the NSAID was recommended by their pharmacist $(15 \%)$. The rest were recommended by friends, relative and TV/radio (Figure 8).

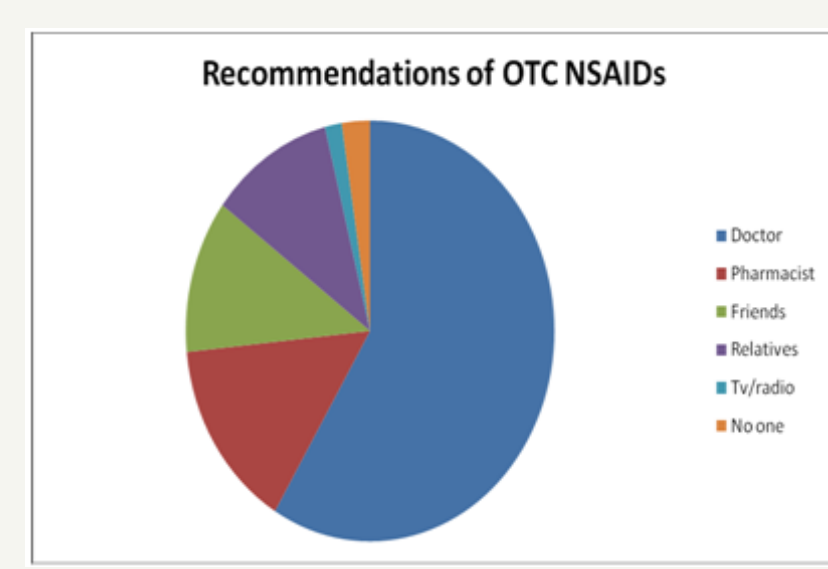

Figure 8: Recommendations of OTC NSAIDs

(No one: participants indicated that no one has recommended NSAID for them i.e. self treatment).

Alarm symptoms reported by participants

Of the total 200 participants, only one was reported to have had blood in stools. The subject was referred immediately to the doctor. Participants' knowledge of NSAIDs use a section in questionnaire asked if participants knew whether the use of NSAIDs was safe for various medical conditions. Of the 200 people surveyed, when asked if they believed that NSAID was safe to be used by people with hypertension, $9(4.50 \%)$ said it is safe, while $31(15.5 \%)$ said it is not safe and $160(80 \%)$. did not know of the hypertensive patients who participated in this study $(n=32), 5(15.6 \%)$, said it is safe while $12(37.5 \%)$ said it is not safe and $15(50 \%)$ did not know about the risks associated with NSAIDs .On average $85 \%$ of the participants did not know if NSAIDs would be harmful to patients with heart problems such as angina, heart failure and heart attack, while nearly $25 \%$ of them said it was not safe with any of the heart problems mentioned above.

When people were asked about the safety of OTC NSAID in asthma, $177(88.5 \%)$ of the total participants did not know whether it is safe or not, $20(10 \%)$ said it is unsafe and $3(1.5 \%)$ people said it is safe. Asthmatic patients $(n=20)$ indicated the following, $3(15 \%)$ said it is not safe and $16(80 \%)$ did not know about the risks associated with NSAIDs.

Of the total 200 customers, $172(86 \%)$ did not know if NSAIDs could be taken or not when people have ulcers and the rest said it is unsafe. Other places where people purchase NSAIDs Most people, 189 of the total 200 participants, reported buying their NSAIDs from the pharmacy. Other than pharmacies, supermarkets were the main places where people bought NSAIDs.

\section{Pharmacist interventions}

Community pharmacists participating in the survey identified a wide range of issues and gave recommendations to customers requesting OTC NSAIDs. The two most frequent types of issues identified, in terms of drug related problems, related to contraindications to the use of OTC NSAIDs and drug interactions. Of the 200 participants surveyed in the pharmacy, 55 participants were found to be using the OTC NSAID inappropriately. The estimated prevalence of inappropriate OTC NSAIDs use in this community is $27.5 \%$ ( $95 \%$ confidence interval: $21.3 \%$ to $33.7 \%$ ). Figure 9 shows some characteristics (health conditions such as Hypertension and Asthma) of the participants with inappropriate OTC NSAIDs use (Figure 9).

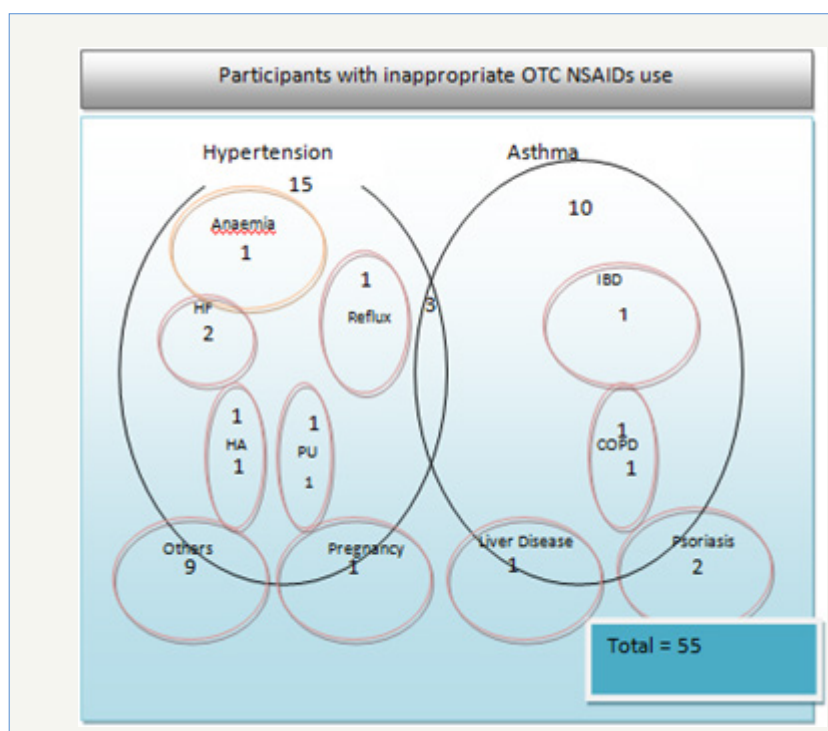

Figure 9: Participants with inappropriate OTC NSAIDs use (HA: heart attack, HF: heart failure, IBD: inflammatory bowel disease, PU: peptic ulcer).

Using the modified DOCUMENT DRP classifications, pharmacists had identified 39(19.5\%) apparent contraindications to the use of NSAIDs, $27(13.5 \%)$ events of drug interactions and $5(2.5 \%)$ cases of duplication Table 3 . Because one patient could have more than one drug-related problem, the events added up to more than 55. Approximately, one in every five customers was advised by pharmacist to change the OTC NSAIDs to a different medication (Table 3).

\section{Multivariate analysis}

Table 4 shows the results of the multivariate logistic regression analysis (Table 4). 
Table 3: Types of drug-related problems.

Number of drug-related problems Drug-related problems subtypes (Percentage) (found in survey)

Drug selection:
Duplication $3(3.7 \%)$
Inappropriate dosage form $2(2.47 \%)$
Drug interaction $26(32 \%)$
Contraindications apparent $39(48.1 \%)$
Wrong drug $1(1.2 \%)$
Other drug selection problems $6(7.4 \%)$

Under-treated or under treated indication:

Condition not adequately treated $4(4.9 \%)$

Total 81(100\%)

\section{Recommendations:}

Dose change $2(3.6 \%)$

Drug change $39(71 \%)$

Medicine not dispensed 12(21.4\%)

Drug formulation change 2(3.6\%)

Total 55(100\%)

\section{Referral required:}

Refer to prescriber 19(9.5\%)

Other referral required 2(1\%)

Total participants $200(100 \%)$

\section{Provision of information:}

Education or counseling session 67(33.5\%)

Total participants $200(100 \%)$

*All other health conditions other than hypertension and asthma e.g. psoriasis, peptic ulcer and pregnancy.

Table 4: Multivariate logistic regression analysis.

\begin{tabular}{|c|c|c|c|}
\hline Variable & Odds Ratio & p-value & $\begin{array}{c}\mathbf{9 5 \%} \text { Confidence } \\
\text { interval }\end{array}$ \\
\hline Ibuprofen + Codeine & 1.95 & 0.1 & 0.87 to 4.39 \\
\hline $\begin{array}{c}\text { Had used NSAID } \\
\text { before }\end{array}$ & 2.81 & 0.016 & 1.21 to 6.53 \\
\hline $\begin{array}{c}\text { Participants with } \\
\text { Asthma }\end{array}$ & 13.8 & $<0.0001$ & 4.24 to 44.9 \\
\hline $\begin{array}{c}\text { Participants with } \\
\text { Hypertension }\end{array}$ & 15.3 & $<0.0001$ & 6.09 to 38.2 \\
\hline
\end{tabular}

The type of medicine requested was initially put into 3 broad groups to improve the stability of the model (due to the small numbers in some categories):

1) Aspirin \pm Codeine;

2) Ibuprofen \pm Codeine and;

3) Other drugs.

It transpired that there were no significant differences in inappropriate use between types of OTC NSAIDs requested, but
Ibuprofen +Codeine showed a trend towards a greater proportion of inappropriate use than the others (not significant). The variables which were most strongly associated with inappropriate use were the presence of asthma and hypertension -if either of these were present, this greatly increased the chance of inappropriate use of the medications. Nearly $70 \%$ of the hypertensive patients were using the NSAIDs inappropriately compared to $60 \%$ amongst asthmatic patients, see table 3 . Similarly, if the subject had taken the medication before, this was also linked to inappropriate use. Other variable found not to be associated (but were initially included in the model), were: number of health conditions, number of medications being taken, age, and gender.

\section{Conclusion}

The prevalence of inappropriate use of OTC NSAIDs in this study was lower than previous studies, yet the prevalence was still significantly high. The study has also demonstrated a lack of knowledge among participants on the risk of OTC NSAIDs use. Study has also revealed the significance role of the pharmacist in preventing drug related problems. It appears that healthcare providers need to have more awareness of the increased inappropriate OTC NSAIDs use in the community. A potential government funded programs for pharmacist lead intervention and education programs may help to minimize the above problem.

\section{Acknowledgement}

This work was part of Master of Clinical Pharmacy at Curtin University supervised by Pascale Ng Cheong Tin, M.Pharm FPS. Many thanks to Dr. Richard Parsons, senior lecturer at Curtin University, for the statistical analysis. Great greetings to Professor Bruce Sunderland for his comments and review of this study.

\section{References}

1. Bleumink GS, Feenstra J, Sturkenboom MC, Stricker BHC (2003) Nonsteroidal anti-inflammatory drugs and heart failure. Drugs 63(6): 525534.

2. FDA (2005) FDA announces series of changes to the class of marketed non-steroidal anti 246 inflammatory drugs (NSAIDs).

3. (2010) Cardiovascular Disease Statistics. Heart Foundation (NHFo) Australia.

4. (2010) 8 Prevalence of upper gastrointestinal conditions and NSAID use 2010. AIHW.

5. ADRAC (2003) ACE inhibitor, diuretic and NSAID: a dangerous combination. Aust Adv Drug React Bull 22: 14-15.

6. Wilcox CM, Cryer B, Triadafilopoulos G (2005) Patterns of use and public perception of over-the254 counter pain relievers: focus on non-steroidal anti-inflammatory drugs. J Rheumatol 32(11): 2218-2224.

7. Laroche ML, Charmes JP, Nouaille Y, Picard N, Merle L (2007) Is inappropriate medication use a major cause of adverse drug reactions in the elderly? Br J Clin Pharmacol 63(2): 177-186.

8. Kovac SH, Houston TK, Weinberger M (2010) Inappropriate Nonsteroidal Anti-Inflammatory Drug Use: Prevalence and Predictors. J Patient Saf 6(2): 86-90.

9. Viktil K, Blix H, Moger T, Reikvam A (2006) Interview of patients by pharmacists contributes significantly to the identification of drugrelated problems (DRPs). Pharmacoepidemiol Drug Saf 15(9): 667-674. 
10. Paulose Ram R, Hirsch R, Dillon C, Gu Q (2005) Frequent monthly use of selected non-prescription and prescription non-narcotic analgesics among U.S. adults. Pharmacoepidemiol Drug Saf 14(4): 257-266.

11. Kovac SH, Saag KG, Curtis JR, Allison J (2008) Association of healthrelated quality of life with dual use of prescription and over-the-counter non-steroidal anti-inflammatory drugs. Arthritis Rheum 59(2): $227-$ 233.

12. Paulose Ram R, Hirsch R, Dillon C, Losonczy K, Cooper M, et al. (2003) Prescription and non271 prescription analgesic use among the US adult population: results from the third National Health and Nutrition Examination Survey (NHANES III). Pharmacoepidemiol Drug Saf 12(4): 315-326.
13. PSA (2010) Australians urged to use caution when using pain relievers 2010.

14. (2010) Promise to document clinical interventions in Australian community pharmacies. Pharmacist: University of Tasmania, Churchill Ave, Hobart, Australia.

15. Australia PSA (2010) Professional Practice Standards Pharmaceutical Society of Australia.

16. Fidell BTL (2007) Using Multivariate Statistics (5 $5^{\text {th }}$ edn). Pearson Education Inc, USA. 